\section{Femtosecond Studies of Electron Dynamics at Interfaces}

\author{
NIEN-HUI GE, ${ }^{\dagger}$,,$\S$ CHUNG M. WONG, ${ }^{t, \neq}$ AND \\ CHARLES B. HARRIS*,t,‡ \\ Department of Chemistry, University of California, \\ Berkeley, California 94720, and Chemical Sciences Division, \\ Ernest Orlando Lawrence Berkeley National Laboratory, \\ Berkeley, California 94720
}

Received December 22, 1998

\section{ABSTRACT}

A delocalized electron at a metal-dielectric interface interacts with the adlayer and spatially localizes or self-traps on the femtosecond time scale into what is termed a small polaron. The dynamics can be studied by two-photon photoemission. Theoretical and experimental analyses reveal the interaction energy and the lattice vibrational mode that mediates electron localization. These results contribute to a fundamental understanding of electron behavior in weakly bonded solids and can lead to a better understanding of carrier dynamics in many different systems, including organic lightemitting diodes.

\section{Introduction}

The study of electronic behavior at interfaces between dissimilar materials constitutes a major research area of fundamental interest and technological significance. For example, hot electrons can induce surface chemical reactions or desorption of adsorbates. ${ }^{1}$ Recent progress in organic light-emitting devices ${ }^{2}$ has heightened the demand for a deeper understanding of electronic processes at metal-organic interfaces where electron trapping, scattering, and the potential barrier at the interface can drastically affect carrier transport properties and the performance of devices. To develop a microscopic understanding of the behavior of electrons in complex media, knowledge of the energy levels associated with interfaces and the dynamics of electrons at the interface is needed.

In this Account we will focus on the study of ultrafast electron dynamics at an interface between a metal sub-

\footnotetext{
Nien-Hui Ge was born in Taipei, Taiwan, in 1968. She received her B.S. degree from National Taiwan University in 1990, her M.S. degree from National Taiwan University in 1992 under the direction of Lian-Pin Hwang, and her Ph.D. from the University of California at Berkeley in 1998 under the direction of Charles B. Harris. She is currently a postdoctoral fellow at the University of Pennsylvania under the direction of Robin M. Hochstrasser.
}

Chung M. Wong was born in Hong Kong in 1971. He received his B.A. degree from Brandeis University in 1994 under the direction of Iu-Yam Chan. He is currently a Ph.D. student at the University of California at Berkeley under the direction of Charles B. Harris.

Charles B. Harris was born in New York, NY, in 1940. He received his B.S. degree from the University of M ichigan in 1963 and his Ph.D. from M .I.T. in 1966, under the direction of F. A. Cotton. Following a postdoctoral fellowship in physics from the Atomic Energy Commission at M.I.T. with the late J ohn C. Slater, he joined the faculty at the University of California, Berkeley in 1967. He currently holds appointments as Professor of Chemistry at U.C. Berkeley and Principle Investigator, Chemical Sciences Division, Lawrence Berkeley National Laboratory. In addition to his work with ultrafast electronic phenomena at surfaces and at interfaces, his research also focuses on organometallic reaction dynamics using femtosecond infrared techniques. strate and a physisorbed dielectric layer. For a more general view of electron dynamics at surfaces, the readers are referred to several review articles. ${ }^{3-7}$ Our goal is to answer two fundamental questions: First, how do the dielectric properties and electronic structure of the overlayer affect the electron dynamics and the interfacial potential that electrons experience? Second, electrons in metals are usually free-electron-like, but electrons in dielectric solids are usually localized. How does the transition of the electronic behavior occur at the interface, and what do we expect the spatial extent of interfacial electrons to be?

The second question is particularly interesting because a long-lasting challenge for both experimentalists and theorists has been the understanding of the polarization interactions of charge carriers with their environment and the resulting polaron formation. Electrons in rigid periodic lattices are delocalized, forming Bloch band states that are free-electron-like. In a deformable lattice, however, the electron-lattice interaction causes an electron to al ways carry with it a self-induced lattice deformation. The composite particle is called a polaron. "Small" polarons, originally conceived by Landau in 1933, involve strong lattice distortion and the wave functions of the carrier are self-trapped to dimensions of a lattice spacing. Their motion between lattice sites can be described as a hopping process, ${ }^{9}$ a behavior observed for charged carriers in many nonmetallic systems. ${ }^{10,11}$ Self-trapped carriers participate in various photochemical and radiation-induced processes, including defect formation ${ }^{12}$ and desorption from surfaces..$^{13}$ In recent years, there has been a surge of renewed interest in small polarons because of the belief that they play an essential role in the properties of new materials such as cuprates and manganites that exhibit, respectively, high-temperature superconductivity and giant magnetoresistance. ${ }^{14}$

Structural and transport properties of small polarons in many systems have been extensively studied experimentally by electron paramagnetic resonance, conductivity measurements, optical spectroscopy, and polarization analysis. ${ }^{10}$ The dynamic process of self-trapping, on the other hand, has only recently been elucidated in real time by utilizing femtosecond lasers. A few measurements have been done by time-resolved transient absorption ${ }^{15}$ and luminescence ${ }^{16}$ methods, revealing the precursor state and the cooling process of self-trapped excitons. M ost studies to date have been performed on bulk materials, whereas very little is known for interfaces. Electron self-trapping and the transition of the electronic behavior near the metal-dielectric interfaces ${ }^{17}$ are fascinating problems in which the effects of reduced dimensionality are important.

To address these questions by investigating properties associated with angstrom scale materials in a well-

\footnotetext{
* To whom correspondence should be addressed.

† University of California, Berkeley.

‡ Ernest Orlando Lawrence Berkeley National Laboratory.

$\S$ Present address: Department of Chemistry, University of Pennsylvania, Philadephia, PA 19104-6323.
} 
Electron Dynamics at Interfaces Ge et al.

(A)

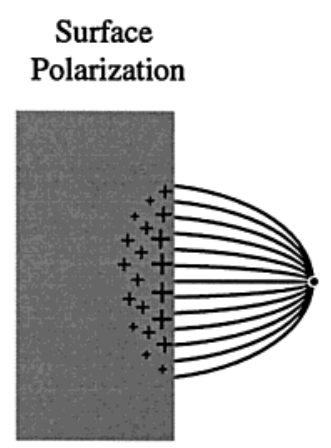

(B)

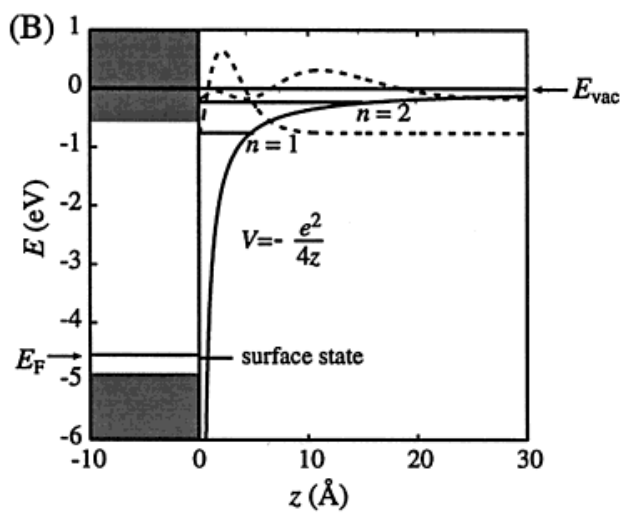

FIGURE 1. (A) An electron near a surface is bound by an image potential which the electron induces by polarizing the material. (B) The square of the hydrogenic wave function is show $n$ for the lowest two image states. The $A g(111)$ band structure for $k_{\|}=0$ is shown in shading. $E_{\mathrm{vac}}$, vacuum energy, $E_{F}$, Fermi energy.

controlled fashion, we applied ultrahigh-vacuum technology and surface preparation methods to grow ultrathin atomic and molecular layers on single crystal metal substrates. To fully explore the dynamics of electrons at interfaces, experimental techniques must provide both band structure specificity and time resolution. In recent years, an experimental technique has been developed to study surface electron dynamics at interfaces by selectively probing the decay of electrons on the surface having a specific parallel momentum to the surface in real time. These electrons, called image state electrons, are particularly sensitive to the interfacial electronic structure since their wave function maximum is located near the surface. In the following sections, we present two photon photoemission (TPPE) results and discuss the electronic tunneling processes and self-trapping of image electrons at alkane/Ag(111) interfaces.

Image States on Bare Metal Surfaces. When an electron is near a surface, e.g., a metal surface, it induces a surface polarization as illustrated in Figure 1A. Classically, this surface polarization can be represented as a positive (image) charge at a distance back from the surface equal to that of the electron from the front. The net result is that the electron feels a Coulomb attraction to the surface-induced polarization or, equivalently, to the image charge. Quantum mechanically, this Coulomb potential, $V(z)=-e^{2} / 4 z$ supports a set of bound energy levels at the surface called image states. ${ }^{18}$ These are illustrated in Figure $1 \mathrm{~B}$ and are the one-dimensional analogues of the
(A)

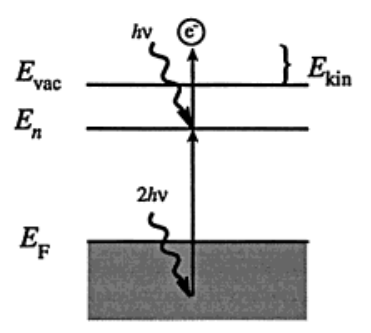

(B)
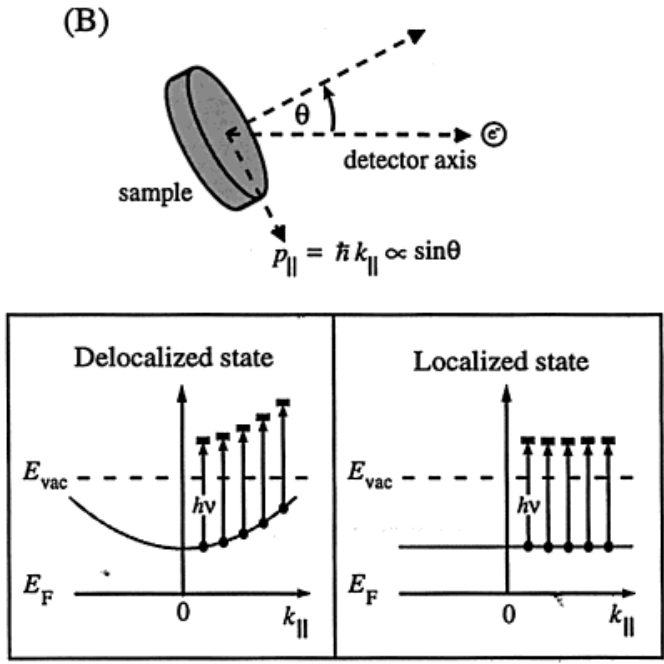

FIGURE 2. (A) Schematic energy diagram for two-photon photoemission (TPPE). (B) Schematic diagram for the photoejection process of angle-resolved TPPE. The dependence of photoelectron kinetic energy on parallel momentum $\hbar k_{\|}$is illustrated for delocalized and localized states.

three-dimensional Rydberg states of the hydrogen atom. In the lowest state, $\mathrm{n}=1$, the expectation value of the electron is only about $3 \AA$ from the surface, and hence, it is very sensitive to changes at the interface from adsorbed layers or other perturbations. The lifetime of image electrons depends mainly on the electron's ability to couple to the substrate empty band structure. Thus, the decay rate of image electrons depends on the overlap of the electronic wave function with the metal substrate.

Femtosecond Angle-Resolved Two-Photon Photoemission. Time and angle resolved TPPE is a technique in which a femtosecond laser excites an electron from occupied states below the Fermi energy $\left(E_{F}\right)$ of a metal to unoccupied image states (Figure 2A). A probe pulse photoionizes the excited electrons above the vacuum level $\left(E_{\mathrm{vac}}\right)$, and the electron kinetic energy $\left(E_{\mathrm{kin}}\right)$ is measured. The difference between $E_{k i n}$ and the probe photon energy yields the binding energy of the intermediate image state $\left(E_{n}\right)$. The decay of the photoelectron signal with increasing time delay between the pump and the probe pulse provides a direct measurement of the intermediate-state lifetime. With the use of ultrashort pulses, dynamics on 10s of femtoseconds can be measured with great sensitivity. Any change in the energy of the intermediate state with time is measured as well.

The band structure at the interface can be determined by measuring the angle dependence of the photoelectron 

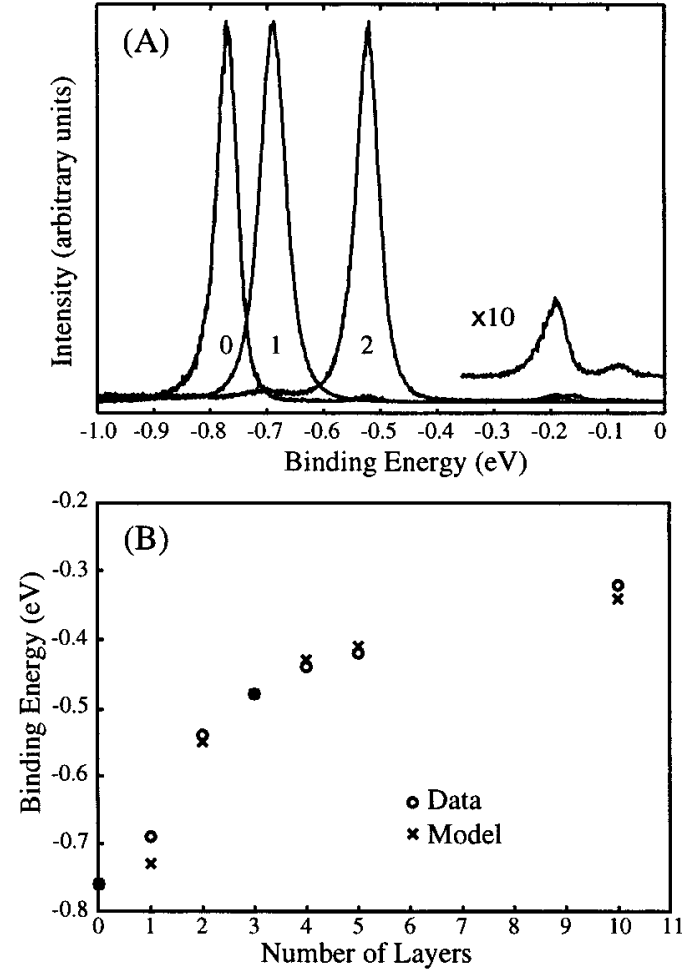

FIGURE 3. (A) TPPE spectra for the image states showing their change in binding energy as a function of $n$-heptane layer thickness on $\mathrm{Ag}(111)$, taken at a pump - probe delay of $160 \mathrm{fs}$. Numbers indicate number of layers. The $n=2,3$ state region is magnified 10 times for the monolayer. (B) Experimental binding energies $(\mathrm{O})$ of the $n=$ 1 states as a function of $n$-octane coverage and a comparison with the results of a dielectric continuum model $(x)$.

kinetic energy (Figure 2B). Photoemission at well-ordered interfaces preserves the electron momentum parallel to the surface $\left(\hbar k_{\|}\right)$which can be experimentally determined by measuring the angle of emission since $\mathrm{k}_{\|}=\left(2 \mathrm{me}_{\mathrm{e}} \mathrm{E}_{\mathrm{kin}}\right)$ $\left.\hbar^{2}\right)^{1 / 2} \sin \theta$ with $\mathrm{m}_{\mathrm{e}}$ being the free-electron mass and $\theta$ the emission angle. ${ }^{19}$ For delocalized electrons behaving like free particles parallel to the interface, the angleresolved TPPE data will exhibit a parabolically dispersive band characterized by an effective mass $\left(\mathrm{m}^{*}\right)$ close to a free-electron value $\left(m_{e}\right)$ :

$$
\mathrm{E}=\mathrm{E}_{0}+\mathrm{E}_{\|}=\mathrm{E}_{0}+\hbar^{2} \mathrm{k}_{\|}^{2} / 2 \mathrm{~m} *
$$

where $E_{0}$ is the onset of the interfacial band at $k_{\|}=0$. On the other hand, spatially localized electrons, which can be roughly thought of as having a very large $\mathrm{m}^{*}$, result in nondispersive flat bands because a localized state is a superposition of many $k_{\|}$plane waves. These two limiting cases are illustrated in Figure 2B.

\section{Electron Tunneling at Metal-Alkane Interfaces}

The image state series persists and becomes less bound with increasing thickness of dielectric layers on $\mathrm{Ag}(111)^{5}$ as shown in the TPPE spectra taken at $0^{\circ}$ emission (Figure 3A). ${ }^{20}$ The change in binding energy correlates to a layerby-layer evolution of the surface potential (Figure 3B).
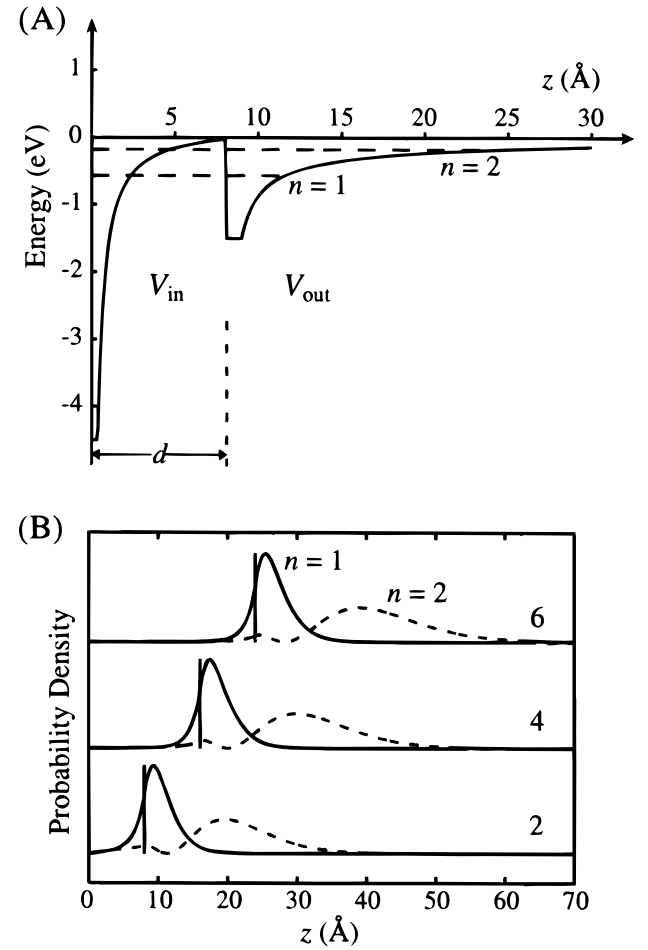

FIGURE 4. (A) Dielectric continuum model potential for a bilayer of $n$-octane. ${ }^{23} \mathrm{~A}$ potential cutoff is imposed at the metal-layer and layer-vacuum interface to avoid the singularity in the potential. The eigenvalues for the $n=1$ and 2 states are show $n$ in the dashed lines. (B) The electron probability density for the $n=1$ and 2 states for 2, 4, and 6 layers of $n$-octane. The vertical lines represent the layer-vacuum boundary.

Low-energy electron diffraction studies have shown that $\mathrm{n}$-alkanes grow ordered layers on the $\mathrm{Ag}(111)$ surface with the carbon-carbon bonds parallel to the surface. ${ }^{21}$ The complex electronic structure of the alkane adlayer outside the metal substrate can be approximated with the dielectric continuum model. ${ }^{22}$ The important parameters used in this model are the layer thickness, $d$, static dielectric constant, $\epsilon$, and the layer electron affinity, $V_{0}$, or the conduction-band minimum. As illustrated in Figure 4A, the potential outside the adlayer in the vacuum region is given by

$\mathrm{V}_{\text {out }}(\mathrm{z}, \mathrm{d})=\frac{-\beta \mathrm{e}^{2}}{4(\mathrm{z}-\mathrm{d})}+\frac{\left(1-\beta^{2}\right) \mathrm{e}^{2}}{4 \beta} \sum_{\mathrm{j}=1}^{\infty} \frac{(-\beta)^{\mathrm{j}}}{\mathrm{z}-\mathrm{d}+\mathrm{jd}}, \quad \mathrm{z}>\mathrm{d}$

where $\beta=(\epsilon-1) /(\epsilon+1)$. The first term is the attractive image potential induced by an excess electron outside an infinite dielectric. The summation describes the influence of the metal substrate. Inside the dielectric, the potential is given as

$$
V_{\text {in }}(z)=-\frac{e^{2}}{4 \epsilon z}+V_{0}, \quad 0<z<d
$$

where the first term is the metal image potential screened by the presence of the dielectric layer. The appearance of the electron affinity level, $V_{0}$, as an additive constant to the potential inside the dielectric provides the simplest 
description of the overlayer band structure. Using parameters, $\epsilon=2.0$ and $\mathrm{V}_{0}=0.2 \mathrm{eV}$, that are reasonably close to those of bulk n-octane, the potential for the bilayer case is shown in Figure 4A. Solving the Schrödinger equation gives $n=1$ binding energies that are in good agreement with the experiment for as many as 10 layers of $n$-octane (Figure 3B). ${ }^{23}$ From the calculated electron probability density (Figure 4B), we see that the electrons tend to be excluded from the layer due to the potential barrier set up by the repulsive electron affinity of the alkane layer. As the layer thickness increases, the coupling to the metal decreases and electrons become less bound.

A more dramatic effect of the layer potential is manifest in the lifetime of image state electrons, particularly the $n$ $=1$ lifetimes that are 32, 155, 1580, and 17600 fs for a bare $\mathrm{Ag}(111)$ surface, monolayer, bilayer, and trilayer of n-heptane on $\mathrm{Ag}$ (111), respectively. ${ }^{23}$ The approximately exponential increase in the $n=1$ lifetime is the result of electron tunneling through the potential barrier of the alkane layer. As discussed in the Introduction, the lifetime of the image state electron is approximately inversely proportional to the image electron density inside the metal substrate. For the simple case of a square potential barrier, an incident wave function toward the barrier will result

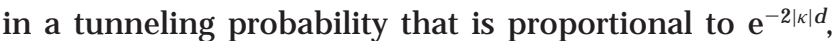
where $\kappa$ is the imaginary part of the wavevector inside the barrier and $d$ is the barrier thickness. For the interfacial image potential as shown in Figure 4A, a model calculation that utilized the WKB approximation to calculate the tunneling probability well reproduced the measured lifetimes. ${ }^{22,23}$ The lifetime of the higher image states ( $n$ > 2) also exhibits an exponential increase as a function of alkane layer thickness on $\mathrm{Ag}(111)$.

In contrast to the results of $n$-alkanes, systems such as neopentane or Xe exhibit very different behavior because these materials possess attractive bulk electron affinities which can provide an interfacial potential well that electrons tend to be drawn into. In fact, the TPPE studies of multilayer $\mathrm{Xe} / \mathrm{Ag}(111)^{24,25}$ indicate quantum confinement effects in the Xe slab. As the Xe layer thickness increases, the $n=2,3$ image states of the bare metal evolve into quantum well states of the layer and become more bound just like a particle in a box. ${ }^{24}$ The drastically different results for $n$-alkane and Xe overlayers clearly show that the dynamics of excess electrons are largely determined by the electron affinity of the adsorbate.

\section{Electron Localization at Metal-Dielectric Interfaces}

While the image states on bare metal surfaces are localized perpendicular to the surface because of the Coulomb attraction for the image charge, parallel to the surface the electrons are delocalized (Figure $2 B$, eq 1 with $E_{0}=E_{n}$ ). On many metal surfaces the effective mass is that of a free electron, i.e., $\mathrm{m}^{*} \approx \mathrm{m}_{\mathrm{e}}{ }^{3}$ The lateral motion of the electron can be drastically altered in the presence of overlayers. In angle-resolved TPPE studies of various alkane/ $\mathrm{Ag}(111)$ interfaces, ${ }^{26}$ besides the parabolically dis-

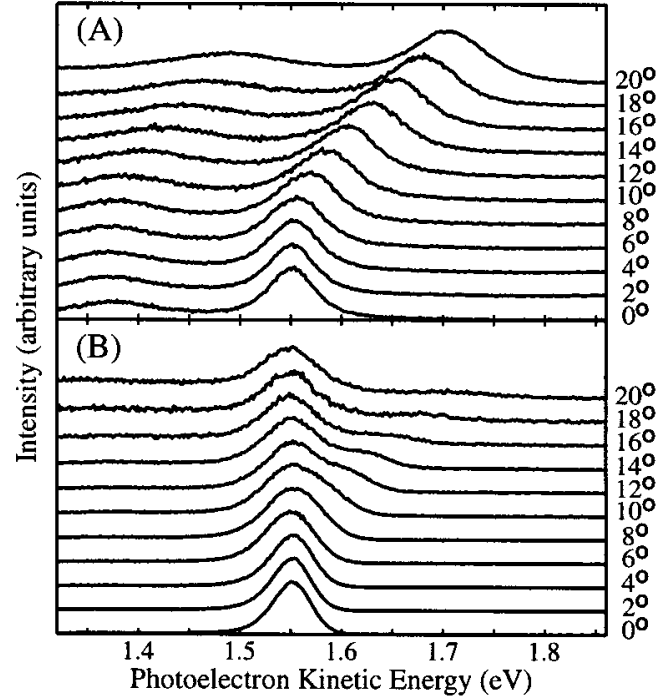

FIGURE 5. (A) Femtosecond angle-resolved spectra taken at $120 \mathrm{~K}$ and a pump - probe delay time of $0 \mathrm{fs}$ and (B) $1670 \mathrm{fs} .{ }^{17}$ Initially the electron is in the delocalized state $\left(m^{*}=1.2 m_{\mathrm{e}}\right)$. The electron then becomes localized within a few hundred femtoseconds. The smaller dispersive feature in $(A)$ is the result of small patches of monolayer interspersed with bilayer.

persing feature (see Figure 2B, left) normally expected for the delocalized $n=1$ image electrons, a nondispersive peak (see Figure 2B, right) was observed and attributed to localized electronic states. These localized features were seen for cyclohexane and various straight-chain alkanes, but not for neopentane. ${ }^{26}$ This observation was correlated with excess electron mobility in nonpolar liquids. ${ }^{27}$

The time scale of the localization process and the mechanism has been further elucidated by a femtosecond time-resolved study. ${ }^{17}$ Figure 5 shows angle-resolved TPPE spectra for bilayer $\mathrm{n}$-heptane at $120 \mathrm{~K}$ taken at two different pump-probe delays. At zero delay time a dispersive feature with $\mathrm{m}^{*}=1.2 \mathrm{~m}_{\mathrm{e}}$ appears (Figure $5 \mathrm{~A}$ ), whereas at a 1670-fs delay this feature is mostly gone and a nondispersive feature dominates the spectra (Figure 5B). These data clearly reveal that the $n=1$ delocalized state appears right after pump pulse excitation, and there is a time delay in the formation of the localized state. Similar behavior is also found on a monolayer and a trilayer. A very interesting aspect of the data is that the localized peak and the delocalized peak are very close in energy at $k_{\|}=$ 0 with a difference $\Delta \mathrm{E}_{0} \leq 10 \mathrm{meV}$. This energy difference is independent of layer thickness and composition (chain length).

The dynamics of these two states were measured at various angles. The localized states exhibit no angle dependence in their 360-fs rise and 1600-fs decay dynamics (Figure $6 \mathrm{~A}$ ), showing that all of the constituting $k_{\|}$plane wave components have the same dynamics, as expected for photoemission from a single state. In contrast, the rise and decay of the $n=1$ delocalized states have strong angle dependence (Figure 6B). We note that the decay times of the delocalized states are of the same order as the rise time of the localized state. This is the case even at different temperatures. Furthermore, both rates become faster as 


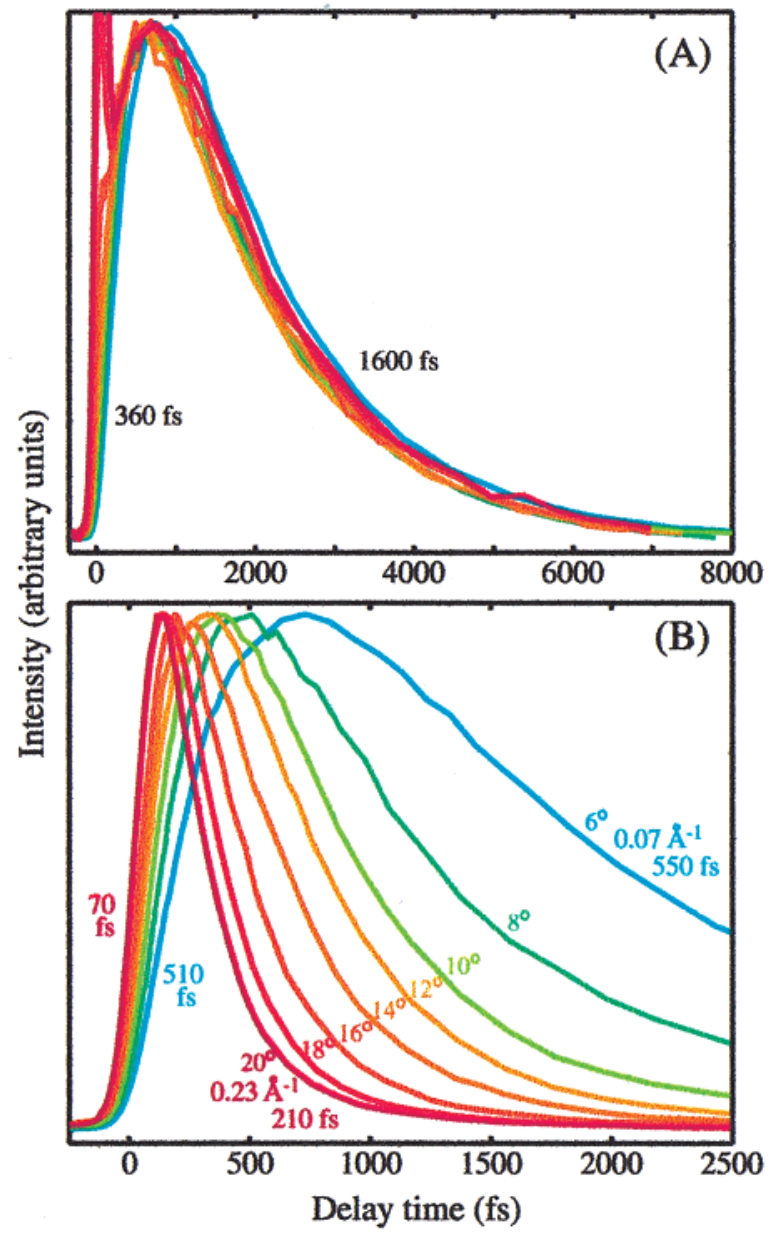

FIGURE 6. Ultrafast time-resolved TPPE traces for (A) localized and (B) delocalized state for bilayer $n$-heptane on $A g(111)$ at various angles and $120 \mathrm{~K} .{ }^{17}$ The extracted rise time and decay time for the delocalized state at $6^{\circ}$ and $20^{\circ}$ as well as the corresponding $k_{\|}$are depicted in (B). The spikes near time zero in (A) for data at high angles come from short-lived electrons on small patches of monolayer.

the temperature is lowered from 120 to $50 \mathrm{~K}$. Such a unique temperature dependence is opposite to the behavior found on other states that do not show localization, such as the bilayer, $\mathrm{n}=2$, state $\mathrm{e}^{20,28}$ and bare surface, $\mathrm{n}=$ 1, state. 20,29 These observations indicate that the delocalized electron primarily decays into the localized state, and the localization time not only depends on its initial parallel momentum but also exhibits a non-Arrhenius temperature dependence. We attribute these results to the self-trapping process responsible for small polaron formation.

Small Polaron Formation. Qualitatively, the degree of localization of carriers in a deformable lattice is determined by a balance of two competing tendencies ${ }^{30}$ as illustrated in Figure 7. Here, curves $\mathrm{V}_{\mathrm{f}}\left(\mathrm{K}_{1,}, \mathrm{Q}\right)$ and $\mathrm{V}_{\mathrm{s}}(\mathrm{Q})$ are associated, respectively, with the delocalized states and the self-trapped state. $\mathrm{Q}$ is the lattice distortion coordinate. Each curve in the $V_{f}$ manifold represents a different $k_{\| \mid}$state with a band energy $E_{\|}\left(E_{\|}=0\right.$ at $k_{\|}=0$, see eq 1$)$. Let us first assume that an electron is localized at a single lattice site without inducing lattice distortion, i.e., along the $\mathrm{Q}$
$=0$ axis. The electron's energy will be at around the band center (point C) because a spatially localized wave packet contains all of the Bloch waves in the band due to the space-momentum uncertainty principle. This localized electron has a tendency to become delocalized by lowering its kinetic energy to reside at the bottom of the band (point F). The cost in energy to keep the electron localized can be estimated from the half-width of the band, B. On the other hand, the localized electron tends to become stabilized through small polaron formation because it can "dig" itself an attractive potential well by polarizing and displacing the atoms surrounding it (point $S, Q \neq 0$ ). If the lattice relaxation energy, $\mathrm{E}_{\mathrm{rel}}$, is large enough such that self-trapping energy $E_{s t} \equiv E_{\text {rel }}-B$ is positive, the selftrapped state will be energetically favorable and a small polaron will form.

The balance between these opposing tendencies depends on the dimensionality of the lattice and the nature and the strength of its coupling with the electron. For a short-range electron-lattice coupling, which is the case for electrons in nonpolar alkane solids, theoretical analysis has shown ${ }^{31-33}$ that localization is energetically favored over delocalization in 1-D, and delocalization is favored in 3-D. In a 2-D system, however, these two tendencies are nearly equal. Thus, two-dimensionality is often called the marginal case $\mathrm{e}^{33,34}$ and results in a small self-trapping energy, $E_{s t}$.

Our data are well-explained by the above 2-D small polaron formation model. The lattice and the molecules that comprise it respond to the presence of an excess electron on the time scale of the lattice and molecular vibrational periods. This is manifest in the time delay between the population of the image state (Figure 5A) and the formation of the localized self-trapped electrons (Figure 5B). The lifetime of the self-trapped electron is $\mathrm{k}_{1^{-}}$ independent (Figure 6A), but is dramatically lengthened with increasing alkane layer thickness. The lifetimes discussed in the previous section on electron tunneling refer to the decay time of the localized state. The tunneling model explains the decay mechanism of the localized state and suggests that the self-trapped electron is localized at the layer-vacuum junction (Figure 4B) rather than at the layer-metal junction. The self-trapping process is associated with interactions between the electron and the topmost plane of the alkane molecules. The small selftrapping energy, as measured by $\Delta \mathrm{E}_{0} \leq 10 \mathrm{meV}$, indicates the existence of a delicate balance between the delocalizing and localizing tendencies in this 2-D system.

Self-Trapping Dynamics. The small polaron model also predicts a momentum-dependent self-trapping time. Inspecting the potential surfaces of the delocalized and selftrapped states (Figure 7), one sees that an energetically favorable pathway to get from $V_{f}$ to $V_{s}$ without first reaching the point $C$ involves thermal activation of the lattice to a configuration in which the energy of the system is the same on both sides, i.e., the crossing point of $V_{f}$ and $V_{s}$. The energy difference between the crossing point and $V_{f}\left(k_{11}\right)$ at $Q=0$ represents the potential barrier or the activation energy $\left(E_{a}\right)$ for self-trapping. The self-trapping 


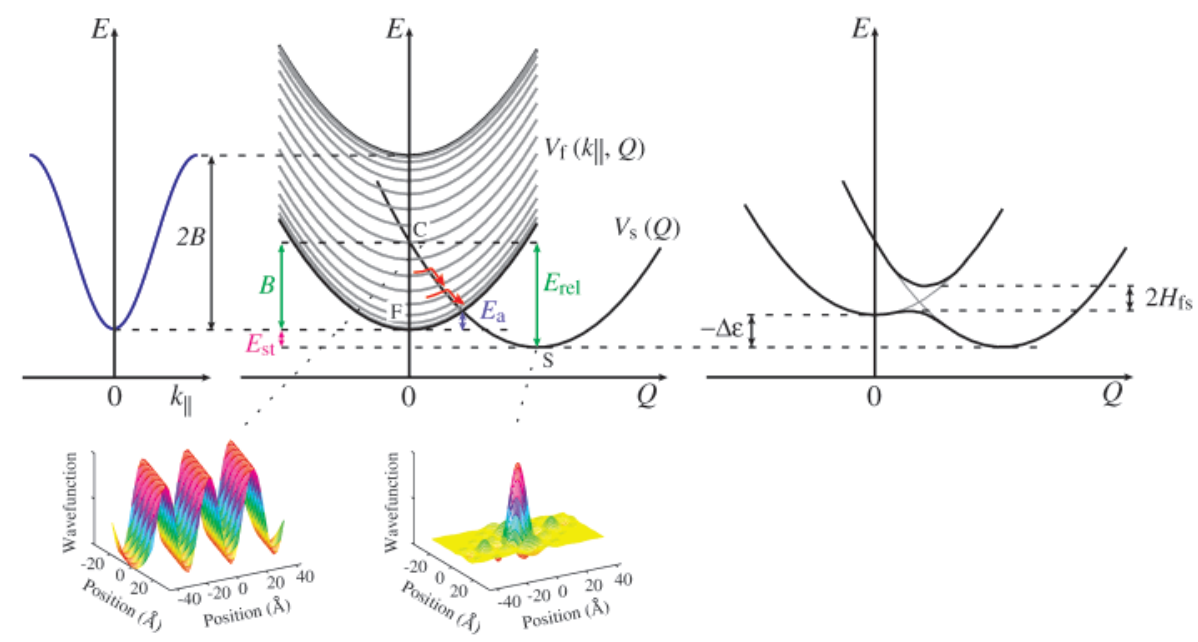

FIGURE 7. Configuration coordinate diagram for self-trapping of an electron. The activation energy of self-trapping $\left(E_{\mathrm{a}}\right)$ for $k_{\|}=0$ is depicted explicitly. Red arrows indicate the classical barrier crossing from the delocalized to the localized state. Schematic illustration of the electron wave function at the $n$-heptane/Ag(111) interface is shown at the bottom. Left: a delocalized state with $k_{\|}=0.22 \AA^{-1}$. Right: a localized state which is assumed to be a 2-D wave packet composed of all $k_{\| \mid}$in the first surface Brillouin zone of the $n$-heptane lattice.

barrier is large at $k_{\|}$, decreases with $k_{\|}$until reaching the point $C$ where there is no barrier, and then turns around to increase with $k_{\|}$. Thus, the self-trapping rate will have a strong dependence on $\mathrm{k}_{\|}$(Figure $6 \mathrm{~B}$ ). On the other hand, the rate at which the self-trapped electrons form will be an averaged sum over self-trapping rates from all $k_{\|}$states (Figure 6A). ${ }^{35}$

The above description of the self-trapping process by the crossing of two potential-energy levels spanned by a generalized nuclear coordinate is analogous to treatments found in the theory for small-polaron hopping ${ }^{9}$ and for electron-transfer reactions. ${ }^{36,37}$ Formally, Holstein smallpolaron theory and Marcus electron-transfer theory are isomorphic. In this analogy, a self-trapping process that starts from an initial state in the $V_{f}$ manifold, having a particular momentum $k_{\|}$and band energy $E_{\|}$, and then proceeds to the self-trapped state at $\mathrm{V}_{\mathrm{s}}$ corresponds to an electron-transfer reaction with exothermicity of $-\Delta \varepsilon=\mathrm{E}_{\mathrm{st}}$ $+\mathrm{E}_{\|}$. Within the displaced harmonic potential approximation and in the classical high-temperature limit where the system has a thermal energy higher than the nuclear vibrational energy, self-trapping occurs through activated barrier crossing and the rate is given by $9,36,37$

$$
\mathrm{k}_{\mathrm{st}}=A \mathrm{e}^{-\left(\Delta \varepsilon+\mathrm{E}_{\mathrm{rel}}\right)^{2} / 4 \mathrm{E}_{\mathrm{rel}} \mathrm{K}_{\mathrm{B}} T}
$$

where $\left(\Delta \varepsilon+\mathrm{E}_{\mathrm{rel}}\right)^{2} / 4 \mathrm{E}_{\text {rel }}$ is the barrier height. Thus, the rate initially increases with $\mathrm{E}_{\|}$and then decreases, reaching the maximum when the exothermicity is equal to the reorganization energy, $-\Delta \varepsilon=\mathrm{E}_{\text {rel }}$. This is referred to as the Marcus inverted region.

The prefactor $A$ in eq 4 depends on the extent of the mixing between the initial and final states at the crossing point, which is determined by the matrix element $\mathrm{H}_{\mathrm{fs}}$ of electronic coupling between the two states (Figure 7). If the nuclei move very slowly such that the nuclear dynamics can be neglected, there is plenty of time spent in the curve-crossing region for electron self-trapping. The reac- tion happens with a high transmission coefficient, and A is equal to the attempt frequency, i.e., the frequency of the harmonic potential well. This is the adiabatic limit which corresponds to electronic coupling being strong enough to ensure that the system follows the lower pathway at all times. The opposite limit, then, is the nonadiabatic limit corresponding to weak electronic coupling. In this limit, lowest order perturbation theory in the electronic coupling can be utilized and $A$ is proportional to $\mathrm{H}_{\mathrm{fs}}^{2}$, which yields the well-known golden rule results.

We obtained the self-trapping rate by subtracting a $\mathrm{k}_{1-}$ independent tunneling rate (previous section) from the delocalized state decay rate. Exothermicity is determined by the photoelectron kinetic energy difference between the delocalized and localized states (see Figure 5). To obtain a good fit of $k_{s t}$ versus $-\Delta \varepsilon$ using the classical theory, however, requires an unreasonable adjustment of the temperature. ${ }^{17}$ The observed non-Arrhenius temperature dependence also suggests the inadequacy of the classical theory. Electrons in a molecular lattice can interact with both intra- and intermolecular vibrations, forming molecular and lattice polarons, respectively. ${ }^{38}$ In our temperature range, the low-frequency intermolecular vibrations can be treated classically, while the highfrequency intramolecular modes are frozen and require a quantum-mechanical treatment. We made the simplest assumption that self-trapping involves a single quantummechanical intramolecular mode with a frequency $\omega_{\mathrm{q}}$ and a reorganization energy $\mathrm{E}_{\mathrm{rq}}$ and all other intermolecular modes are classical modes with reorganization energy $E_{r c}$. Without knowing the magnitude of the electronic coupling between the delocalized and self-trapped states, it is hard to determine whether the process is adiabatic or nonadiabatic. Therefore, we analyzed the data in terms of a recently developed approach ${ }^{39}$ in which the path integral technique is utilized to sum over all perturbation orders in electronic coupling. This formalism is valid from the 

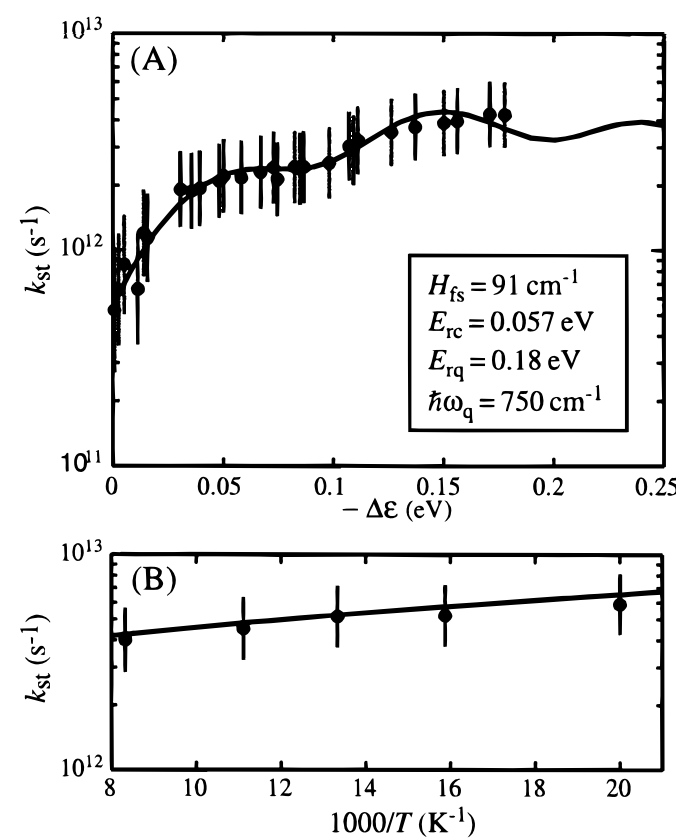

FIGURE 8. (A) A logarithmic plot of the self-trapping rate of the delocalized state versus exothermicity for a bilayer at $120 \mathrm{~K}^{17}$ The $95 \%$ confidence limit for each data point is indicated by the vertical line. The solid line was computed by quantum electron transfer theory ${ }^{39}$ with the parameters listed in the figure. (B) Temperature dependence of self-trapping rates for a bilayer at $\left.18^{\circ} k_{||}=0.21 \AA^{-1}\right)_{.17}^{17}$ The solid line was computed with $-\Delta \varepsilon=0.14 \mathrm{eV}$ and the same parameters as in $(A)$.

nonadiabatic to the adiabatic regimes with its first order term coinciding exactly with the exact quantum mechanical expression for the nonadiabatic rate.

Figure $8 \mathrm{~A}$ shows the result of our analysis. ${ }^{17}$ The parameters $E_{r c}$ and $E_{r q}$ extracted from the fit are consistent with the interaction energies of lattice and molecular polarons in organic molecular solids. ${ }^{38}$ The energy of $\hbar \omega_{\mathrm{q}}$ suggests that self-trapping involves the methylene rocking mode of $n$-heptane molecules. ${ }^{40}$ Note that the first-order term in the path integral accounts for nearly $96 \%$ of the fit in Figure 8A. Therefore, the self-trapping process at 120 $\mathrm{K}$ can be well-described by a nonadiabatic theory. Figure $8 \mathrm{~B}$ shows that the non-Arrhenius temperature dependence of the self-trapping rate at $-\Delta \varepsilon=0.14 \mathrm{eV}$ is reproduced with no additional adjustment of parameters. The firstorder term accounts for only $83 \%$ of the rate for the data point at $50 \mathrm{~K}$.

\section{Conclusions}

In this Account, we have discussed the application of timeand angle-resolved TPPE in the study of excess electron dynamics at metal-dielectric interfaces on the femtosecond time scale. First, we monitored the layer-by-layer evolution of the interfacial electronic structure and dynamics. Such a study provides information on the nature of the interfacial potential. It is found that the electron affinity of the overlayer material strongly affects the dynamics and energies of the interfacial excited states. The formation of a tunneling barrier or potential well at the interface due to the presence of the adlayer plays a very important role in determining the spatial distribution of the interfacial electrons as well as the electronic coupling across the interface.

We have also shown that an initially delocalized electron at the metal-alkane interface undergoes 2-D selftrapping within a few hundred femtoseconds. Our analysis shows that self-trapping involves inter- and intramolecular vibrational modes of the overlayer and the non-Arrhenius temperature dependence is a result of a strong quantum contribution from the intramolecular modes. These results for a model interface contribute to the fundamental understanding of electron behavior at the interface between metals and dielectric solids. For example, similar localized states may exist at metal-polymer interfaces important in organic LEDs and contribute to their electronic properties.

The ability of time-resolved TPPE to completely determine the time evolution of the quantum state of excited electrons at interfaces provides detailed experimental information that is hardly accessible by other techniques. In addition to picking out the energy and parallel momentum of the state by measuring the kinetic energy and emission angle of photoelectrons, one can also spinresolve the electrons ${ }^{41}$ determine the phase relaxation by an interferometric scheme, ${ }^{42}$ and study the motion of electron wave packets by coherent excitation. ${ }^{43}$ The versatile capabilities of this technique will undoubtedly open avenues in the understanding of electronic behavior in a wide variety of systems.

We gratefully acknowledge Dr. Robert L. Lingle, Jr., Jason D. McNeill, Robert E. Jordan, and Kelly J. Gaffney, who wereinvolved in many of the experiments that are discussed and referenced in this paper. We particularly thank Dr. Xueyu Song for very helpful discussions and his provision of the electron transfer rate formula. ${ }^{39}$ This work was supported by the Director, Office of Energy Research, Office of Basic Energy Sciences, Chemical Sciences Division, U.S. Department of Energy, under Contract no. DE-AC0376SF00098. The authors acknowledge NSF support for specialized equipment used in some experiments described herein.

\section{References}

(1) Avouris, P.; Walkup, R. E. Fundamental Mechanisms of Desorption and Fragmentation induced by Electronic Transitions at Surfaces. Annu. Rev. Phys. Chem. 1989, 40, 173-206.

(2) Sheats, J. R.; Antoniadis, H.; Hueschen, M.; Leonard, W.; Miller, J .; Moon, R.; Roitman, D.; Stocking, A. Organic Electroluminescent Devices. Science (Washington, D.C.) 1996, 273, 884-888.

(3) Fauster, T.; Steinmann, W. Two-Photon Photoemission Spectroscopy of Image States. In Photonic Probes of Surfaces; Halevi, P., Ed.; Elsevier: Amsterdam, 1995; pp 347-411.

(4) Haight, R. Electron Dynamics at Surfaces. Surf. Sci. Rep. 1995, $21,277-325$

(5) Harris, C. B.; Ge, N.-H.; Lingle, R. L., J r.; McNeill, J . D.; Wong, C. $M$. Femtosecond Dynamics of Electrons on Surfaces and at Interfaces. Annu. Rev. Phys. Chem. 1997, 48, 711-744.

(6) Petek, H.; Ogawa, S. Femtosecond Time-Resolved Two-Photon Photoemission Studies of Electron Dynamics in Metals. Prog. Surf. Sci. 1997, 56, 239-310.

(7) Osgood, R. M. J .; Wang, X. Image States on Single-Crystal Metal Surfaces. In Solid State Physics; Ehrenreich, H., Spaepen, F., Eds. Academic Press: New York, 1998; Vol. 51, pp 1-80.

(8) Landau, L. D. Phys. Z. Sowjetunion 1933, 3, 664. 
(9) Holstein, T. Studies of Polaron Motion, Part II. The "Small" Polaron. Ann. Phys. (Leipzig) 1959, 8, 343-389.

(10) Song, K. S.; Williams, R. T. Self-Trapped Excitons, 2nd ed: Springer-Verlag: Berlin, 1996.

(11) Shluger, A. L.; Stoneham, A. M. Small Polarons in Real CrystalsConcepts and Problems. J. Phys.: Condens. Matter 1993, 5, 30493086.

(12) Hayes, W.; Stoneham, A. M. Defects and Defect Processes in Nonmetallic Solids; Wiley: New York, 1985.

(13) Burns, A. R.; Stechel, E. B.; J ennison, D. R., Eds. Desorption Induced by Electronic Transitions; Springer: Berlin, 1993.

(14) Lanzara, A.; Saini, N. L.; Brunelli, M.; Natali, F.; Bianconi, A.; Radaelli, P. G.; Cheong, S.-W. Crossover from large to small polarons across the metal-insulator transition in manganites. Phys. Rev. Lett. 1998, 81, 878-881.

(15) Iwai, S.; Tokizaki, T.; Nakamura, A.; Tanimura, K.; Itoh, N.; Shluger, A. One-Center Small Polarons as Short-Lived Precursors in SelfTrapping Processes of Holes and Electron-Hole Pairs in Alkali lodides. Phys. Rev. Lett. 1996, 76, 1691-1694.

(16) Tomimoto, S.; Nansei, H.; Saito, S.; Suemoto, T.; Takeda, J .; Kurita, S. Femtosecond dynamics of the exciton self-trapping process in a quasi-one-dimensional halogen-bridged platinum complex. Phys. Rev. Lett. 1998, 81, 417-420.

(17) Ge, N.-H.; Wong, C. M.; Lingle, R. L., J r.; McNeill, J . D.; Gaffney, K. J .; Harris, C. B. Femtosecond Dynamics of Electron Localization at Interfaces. Science (Washington, D.C.) 1998, 279, 202-205.

(18) Echenique, P. M.; Pendry, J . B. Theory of Image States at Metal Surfaces. Prog. Surf. Sci. 1990, 32, 111-172.

(19) Smith, N. V.; Kevan, S. D. Introduction. In Angle-Resolved Photoemission: Theory and Current Applications; Kevan, S. D., Ed.; Elsevier: Amsterdam, 1992; pp 1-14.

(20) Ge, N.-H. Ultrafast Studies of Electron Dynamics at MetalDielectric Interfaces. Ph.D. Thesis, University of California at Berkeley, 1998.

(21) Firment, L. E.; Somorjai, G. A. Low-Energy Electron Diffraction Study of the Surface of Thin Crystals and Monolayers of Normal Paraffins and Cyclohexane on the Ag(111) Crystal Surface. J. Chem. Phys. 1978, 69, 3940-3952.

(22) Cole, M. Electronic Surface States of a Dielectric Film on a Metal Substrate. Phys. Rev. B: Solid State 1971, 3, 4418-4422.

(23) Lingle, R. L., J r.; Ge, N.-H.; J ordan, R. E.; McNeill, J . D.; Harris, C. B. Femtosecond Studies of Electron Tunneling at Metal-Dielectric Interfaces. Chem. Phys. 1996, 205, 191-203; Chem. Phys. 1996 208, 297-298.

(24) McNeill, J. D.; Lingle, R. L., J r.; J ordan, R. E.; Padowitz, D. F.; Harris, C. B. Interfacial Quantum Well States of $\mathrm{Xe}$ and $\mathrm{Kr}$ Adsorbed on Ag(111). J. Chem. Phys. 1996, 105, 3883-3891.

(25) McNeill, J . D.; Lingle, R. L., J r.; Ge, N.-H.; Wong, C. M.; J ordan, R. E.; Harris, C. B. Dynamics and Spatial Distribution of Electrons in Quantum Wells at Interfaces Determined by Femtosecond Photoemission Spectroscopy. Phys. Rev. Lett. 1997, 79, 46454648.

(26) Lingle, R. L., J r.; Padowitz, D. F.; J ordan, R. E.; McNeill, J . D.; Harris, C. B. Two-Dimensional Localization of Electrons at Interfaces. Phys. Rev. Lett. 1994, 72, 2243-2246.

(27) Allen, A. O. Drift Mobilities and Conduction Band Energies of Excess Electrons in Dielectric Liquids; NBS Report No. NSRDSNBS 58; U.S. Department of Commerce, Washington, D.C., 1976.
(28) Interestingly, the decay rate of the $n=2$ state for a bilayer $n$-heptane not only decreases with decreasing temperature but also exhibits a linear dependence on $E_{\mid 1}$. The mechanism for this $k_{\mathrm{n}}{ }^{2}$ dependence is currently under investigation.

(29) Knoesel, E.; Hotzel, A.; Wolf, M. Temperature Dependence of Surface State Lifetimes, Dephasing Rates and Binding Energies on $\mathrm{Cu}(111)$ Studied with Time-Resolved Photoemission. J. Electron Spectrosc. Relat. Phenom. 1998, 88-89, 577-584.

(30) Toyozawa, Y. Electrons, Holes and Excitons in Deformable Lattice. In Relaxation of Elementary Excitations; Kubo, R., Hanamura, E., Eds.; Springer-Verlag: Berlin, 1980; pp 3-18.

(31) Emin, D.; Holstein, T. Adiabatic Theory of an Electron in a Deformable Continuum. Phys. Rev. Lett. 1976, 36, 323-326.

(32) Toyozawa, Y.; Shinozuka, Y. Stability of an Electron in Deformable Lattice-Force Range, Dimensionality and Potential Barrier. J. Phys. Soc. J pn. 1980, 48, 472-478.

(33) Ueta, M.; Kanzaki, H.; Kobayashi, K.; Toyozawa, Y.; Hanamura, E. Excitonic Processes in Solid; Springer-Verlag: Berlin, 1986.

(34) Sumi, H. Peculiarities in Exciton-Polaron Formation and SelfTrapping in Low Dimensions. In Excitonic Processes in Condensed Matter, Proc. SPIE 2362; Singh, J ., Ed.; SPIE: Bellingham, WA, 1995; pp 108-119.

(35) In fact, this picture is supported by the wavelength dependence of the rise time of the localized state. With a 300-nm pump600$\mathrm{nm}$ probe, a wide range of $k_{1}\left(0-0.4 \AA^{-1}\right)$ states can be populated, and they can all decay into the localized state. This results in a rise time of $360 \mathrm{fs}$ (Figure 6A). Selectively populating a narrower range of high $k_{1}$ states with a faster self-trapping rate should reduce the rise time. This prediction is borne out by the results obtained for a 335-nm pump670-nm probe by which only states with $k_{\|} 0.15-0.17 \AA^{-1}$ can be populated, resulting in a faster rise by a factor of 2 .

(36) Marcus, R. A.; Sutin, N. Electron Transfers in Chemistry and Biology. Biochim. Biophys. Acta 1985, 811, 265.

(37) Ulstrup, J. Charge-Transfer Processes in Condensed Media; Springer-Verlag: Berlin, 1979.

(38) Silinsh, E. A.; Cápek, V. Organic Molecular Crystals: Interaction, Localization, and Transport Phenomena; American Institute of Physics: New York, 1994.

(39) Stuchebrukhov, A. A.; Song, X. Y. Quantum Effects in ElectronTransfer Reactions with Strong Electronic Coupling. J. Chem. Phys. 1994, 101, 9354-9365.

(40) Snyder, R. G. Vibrational Study of the Chain Conformation of the Liquid $n$-Paraffins and Molten Polyethylene. J. Chem. Phys. 1967, 47, 1316.

(41) Aeschlimann, M.; Bauer, M.; Pawlik, S.; Weber, W.; Burgermeister, R.; Oberli, D.; Siegmann, H. Ultrafast Spin-Dependent Electron Dynamics in FCC Co. Phys. Rev. Lett. 1997, 79, 5158-5161.

(42) Ogawa, S.; Nagano, H.; Petek, H.; Heberle, A. Optical Dephasing in $\mathrm{Cu}(111)$ Measured by Interferometric Two-Photon TimeResolved Photoemission. Phys. Rev. Lett. 1997, 78, 1339-1342.

(43) Hofer, U.; Shumay, I. L.; Reuss, C.; Thomann, U.; Wallauer, W. Fauster, T. Time-Resolved Coherent Photoelectron Spectroscopy of Quantized Electronic States on Metal Surfaces. Science (Washington, D.C.) 1997, 277, 1480-1482. 